\title{
L'intérêt des mucoprotéines en pathologie digestive
}

L'exploration physico-chimique du serum sanguin se perfectionne, sans pour autant faciliter la tâche du praticien, avide de disposer de tests fidèles et simples; or il n'existe, par exemple, aucune relation régulière entre les modifications des épreuves qualitatives usuelles et les alterations de la courbe électrophorétique. C'est pourquoi le dosage d'une nouvelle fraction du serum, mise en evidence dès 1948 par P. Winzler, et étudiée de façon approfondie par E. M. Greenspan, mérite de retenir $\Gamma$ attention.

Les mucoprotéines ne représentent pas un corps nettement défini, mais une fraction des glycoprotéines, faisant partie essentiellement des globulines- $01 / 8$ (Winzler). Leur caractère chimique est determine par leur precipitation à Гacide phospho-tungstique, après que les autres protéines du serum ont été éliminées par l'acide perchlorique. Leur taux normal dans le serum s'établit entre 60 et $65 \mathrm{mg} \mathrm{p}$. $100 \mathrm{ml}$ avec des valeurs extremes de 40 et $75 \mathrm{mg} \mathrm{p} .100 \mathrm{ml}$ et peut subir, dans les conditions pathologiques, des variations s'étendant de 15 à $300 \mathrm{mg} \%$. Grosso modo, selon Greenspan, ce taux subit Tinfluence depressive des functions hépatiques, tandis que les proliferations cellulaires (in-flammatoires ou néoplasiques) Taugmentent.

Deux groupes de cliniciens français (A. Varay et M. Masson, de

308

\section{Editorial}

Paris: Acta gastro-enterologica belgica de février 1958, p. 251, et Presse Médicale du 9 juillet 1960, p. 1323; M. Gírard et 4 collabora-teurs, de Lyon: Journal de Médecine de Lyon du 5 décembre 1961, p. 1887) viennent de faire part de leur experience pratique des résul-tats obtenus avec le dosage, simple et facile, des mucoprotéines. C'est dans les affections hépatiques que le dosage des mucoprotéines est le plus précieux en clinique. Les hepatites ínfectíeuses s'accompagnent constamment d'une baisse des mucoprotéines (moyenne:

44.6 $\mathrm{mg} \%$ ), la normalisation correspondant avec la guérison; quand en presence de ce diagnostic probable, on trouve une valeur élevée, il faut suspecter une cholangite, un cancer du foie ou une obstruction. Dans la cirrhose, les chiífres sont moins significatifs (moyenne: $55 \mathrm{mg} \%$ ), bien que des taux très bas (15 mg\%) soient d'un pronostic severe; de nouveau là, des chiffres supérieurs à la normale doivent faire reconsidérer le diagnostic. L-Iiypo-mucoprotéínémíe est done un excellent test de deficit de la cellule hépatique. Les ictères mécaniques, d'origine extra-hépatique, s'accompagnent d'un taux élevé de mucoprotéines, de même que le cancer du foie, surtout métastasique (plus de $200 \mathrm{mg} \%$ ). Certains taux d'apparence contradictoire (taux normaux) s'expliquent par la pbase de passage de la cirrhose à l'hépatome, ou par une insuffisance parenchymateuse ajoutée à l'obstacle extérieur.

Enfin, selon Gírard, le dosage des mucoprotéines subit les mêmes modifications dans Vascíte: taux constamment abaissés (moyenne: 
$31.7 \mathrm{mg} \%$ ) en presence de cirrhose, toujours très élevés (moyenne:

$136 \mathrm{mg} \%$ ) dans Гascite carcinomateuse.

Le cancer en general produít une hypermucoprotidémíe, et cette constatation doit faire toujours rechercher une dégénérescence ma-ligne. Ce fait si constant peut contribuer au test thérapeutique d'un ulcère suspect: si celui-ci coincide avec un taux élevé de mucoprotéines (par suite de phénomènes inflammatoires), le retour à la normale après traitement d'épreuve est un argument important en faveur de la bénignité de $\Gamma$ ulcère.

Signalons accessoirement que les cholêcystítes chroníques n'imposent pas de variation au taux des mucoprotéines (qui s'élèvent en cas d'in-fection aiguë); de même avec la recto-côlíte ulcérohémorragique, où la mucoprotidémie dépasse $100 \mathrm{mg} \%$ en phase de poussée evolutive, pour se normaliser en période de latence.

Voici done des nouvelles possibilités venant enrichir la «batterie» de nos moyens d'exploration, en particulier dans le diagnostic parfois si troublant des ictères. M. D. 